\title{
An Efficient LSDM Lighting Control Logic Design on Lighting Control System
}

\author{
Sung-Il Hong, Chi-Ho Lin \\ Schools of Computer, Semyung University \\ 65- Semyung-ro, Jecheon, Chungbuk, Korea \\ megadriver@hanmail.net, ich410@semyung.ac.kr
}

\begin{abstract}
In this paper, we propose an efficient LSDM lighting control logic design on lighting control system. The proposed LSDM lighting control logic is designed according to the operating conditions by the divide as the signal control part for I/O data bus and the timer/counter part for clock signal control. Also, the control logic is transmitted to MCU through a data bus by the environmental information detected from each sensor node. The propose LSDM lighting control logic was measured to power loss rate of the control logic in order to demonstrate the efficiency by applied to the control system. And it was proves that be effective to overall power consumption reduction.
\end{abstract}

Keywords - Control logic, LSDM, Lighting control, Signal control, Power dissipation, MCU

\section{INTRODUCTION}

The field of lighting design in the 21 st century is being developed based of intelligence and automation technology because the LED lighting device performance was improved quickly in recent years. The LED lighting device needs to the control logic for LED emitting. The LED Lighting is common to light by configure to one module the multiple LED. The power consumption of LED is affects to the life of the LED lighting device by changing the internal temperature of the LED through operating of the control logic. Also, the LED lighting control system should be provides exceptional identification skills of things and high color rendering by considering the energy savings [1-6].

The existing lighting device is not complicated control circuit design because to implement of the simple on-off function according to operate of the sensor. But, the existing lighting control logic for the LSDM (LED streetlight dot-matrix module) control has to problems when operating using each different device drivers and control programs. The lighting control logic for LSDM is sensitive device that directly affects that light output of LED, reliability, efficiency and life. Also, the LED lighting device is required design using $\mathrm{MCU}$, because to available to the control system that can be controlled many features and operated. [7-8].

The current flowing through the LED is determined the brightness, and the internal power consumption will change the internal temperature of the LED. Therefore the lighting control logic is sensitive devices that are related LED's brightness, reliability, efficiency, long life, etc., because the operating temperature affects the life of the LED. The LED lighting control logic was various studies performed for have optimal performance about the system configuration or the circuit. However, these research is lacking when compared to the technology for reflect optimal design with modeling of the LSDM lighting control logic. The existing street lighting control system has been operated by design as form provided ability to distinguish objects only to pedestrians or motorists than the light functionality. As a result, it needs the design of the lighting device control system for functional that can ensure the safety of the road higher with reminded the attention of pedestrians by the reminder due to added of a variety add-ons. In this paper for troubleshooting, we propose design of an efficient LSDM lighting control logic for lighting control system using the MCU by the compatibility of the devices and the considering scalability.

\section{LSDM LIGHTING CONTROL LOGIC}

Into ATmega128 is downloaded to your system by compile the lighting control program created for the control of LSDM with the kernel and the device drivers and the file systems. The LSDM lighting control logic is used to control of the LSDM by configure as the decoders, the drivers, the latches, shift registers.

Figure 1 show the configuration of the LSDM lighting control systems. In this paper, efficient control logic for the LSDM operation is designed by configure the LSDM as signal controller, timer and counter parts. The LSDM control signal be to the LSDM lighting control by operate to the control logic with the program execution. The LSDM lighting control logic was designed divided as the signal control part for I/O data bus, the timer/counter part for clock signal control according to operating conditions.

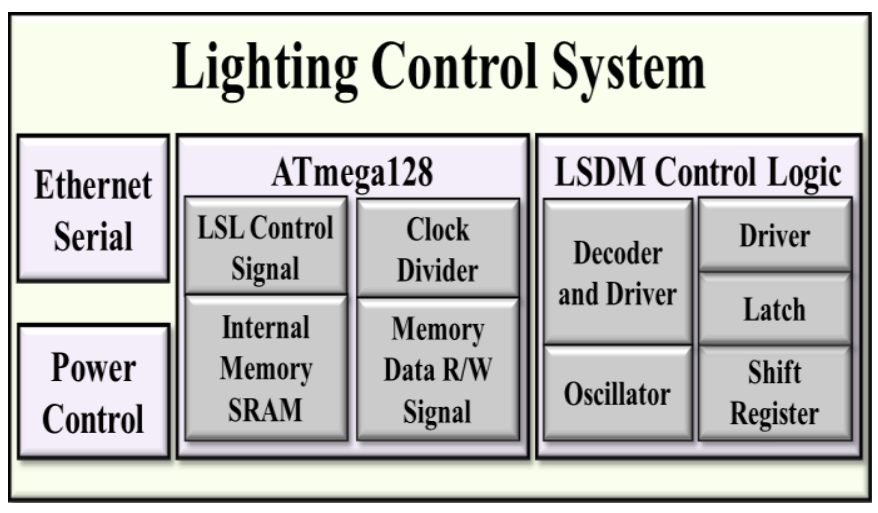

Figure 1. LSDM Lighting control system 
Figure 2 is show to the configuration of the LSDM lighting control logic. The control logic is used decoder for select the lighting line by considering expansion of the LSDM. The I/O of the lighting control data use the latch for controlled. Also, the control logic be to output the lighting data as LSDM using the driver the vertically and horizontally. The oscillator is designed to provide clock synchronization on all circuits using the internal oscillator circuit of ATmega128. The enable is received 1 from ATmega 128 for data output by repeat the latch process after a certain period of time. The R-Data and G-Data used with data for lighting of LSDM. The A0 to A2 of 3-bit address lines are designed to be line selection of LSDM by connect serial as the LSDM of up to maximum eight. It is output the lighting line selection signal of LSDM, because the LSDM I/O data signal controller can be controlled by selecting that LSDM connected as serial by adding a 3-bit decoder. In this case, the LSDM will light up by shift of the lighting data values using a shift register. The anode and the cathode driver are output as LSDM by receiving the lighting data signal of the rows and columns.

Figure 3 show to the configuration default value the optimization register. The control logic is used the output until port $\mathrm{C}$ from port A on ATmega128 for data processing. And, it uses port $\mathrm{G}$ for controlling using address latch signal and $\mathrm{R} / \mathrm{W}$ strobe signal of memory. The signal of the $\mathrm{R} / \mathrm{W}$ signal about to memory access rough to the $\mathrm{I} / \mathrm{O}$ buffer, and the address latch etc. is to light up the LSDM. A port that is used at the I/O of the lighting data signals for LSDM lighting control can be the individual bits control when used as general-purpose I/O ports. Therefore, it is to be read/write to lighting data of LSDM using the PORTx data register (Data Register) that corresponds to the output, and the DDRx (Data Direction Register) that set the direction of $\mathrm{I} / \mathrm{O}$.

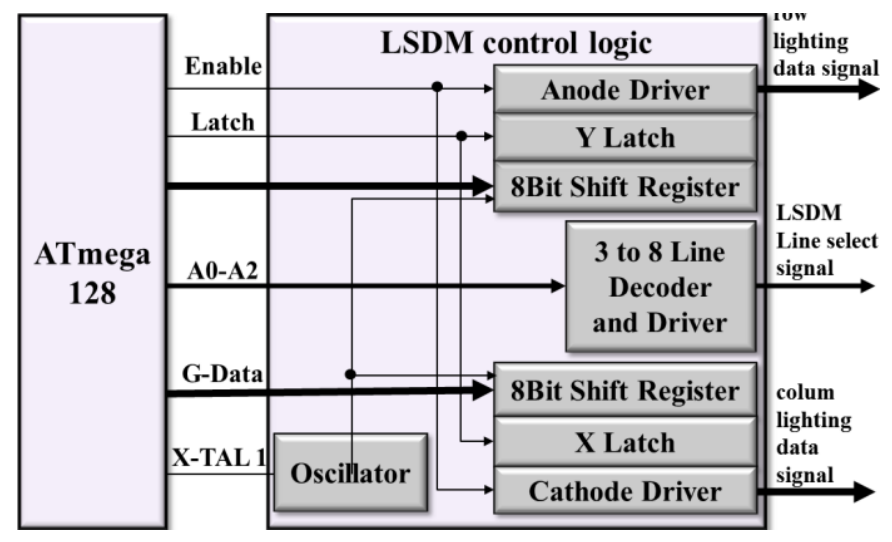

Figure 2. LSDM lighting control Logic

\begin{tabular}{|c|c|c|c|c|c|c|c|c|c|}
\hline \multirow[t]{4}{*}{ DDRx } & & DDRx 7 & DDRx6 & DDRx 5 & DDRx4 & DDRx3 & DDRx2 & DDRxl & DDRx0 \\
\hline & $\begin{array}{l}\text { Read/ } \\
\text { write }\end{array}$ & $\mathrm{R} / \mathrm{W}$ & $\mathrm{R} / \mathrm{W}$ & $\mathrm{R} / \mathrm{W}$ & $\mathrm{R} / \mathrm{W}$ & $\mathrm{R} / \mathrm{W}$ & $\mathrm{R} / \mathrm{W}$ & $R / W$ & $\mathrm{R} / \mathrm{W}$ \\
\hline & $\begin{array}{l}\text { Initial } \\
\text { value }\end{array}$ & 0 & 0 & 0 & 0 & 0 & 0 & 0 & 0 \\
\hline & bit & 7 & 6 & 5 & 4 & 3 & 2 & 1 & 0 \\
\hline \multirow[t]{3}{*}{ PORTx } & & $\mathrm{Px} 7$ & Px6 & Px5 & Px4 & Px3 & Px2 & Pxl & PxO \\
\hline & $\begin{array}{l}\text { Read/ } \\
\text { write }\end{array}$ & $\mathrm{R} / \mathrm{W}$ & $\mathrm{R} / \mathrm{W}$ & $R / W$ & $\mathrm{R} / \mathrm{W}$ & $\mathrm{R} / \mathrm{W}$ & $R / W$ & $R / W$ & $\mathrm{R} / \mathrm{W}$ \\
\hline & $\begin{array}{l}\text { Initial } \\
\text { value }\end{array}$ & 0 & 0 & 0 & 0 & 0 & 0 & 0 & 0 \\
\hline
\end{tabular}

TABLE 1. PORTX DATA Register VAlue

\begin{tabular}{|c|c|c|c|c|}
\hline \multirow{4}{*}{ DDRx } & \multicolumn{4}{|c|}{ PORTx } \\
\cline { 2 - 5 } & $\sim$ row[1] & $\sim$ row[2] & $\sim$ row[3] & $\sim$ row[4] \\
\hline \multirow{7}{*}{ 0xff } & 0xff & 0xdd & 0x01 & 0xee \\
\cline { 2 - 5 } & 0x01 & 0x55 & 0xff & 0xaa \\
\cline { 2 - 5 } & 0xff & 0x55 & 0x80 & 0xaa \\
\cline { 2 - 5 } & 0x80 & 0x55 & 0xff & 0xaa \\
\cline { 2 - 5 } & 0xff & 0x55 & 0x01 & 0xaa \\
\cline { 2 - 5 } & 0x01 & 0x55 & 0xff & 0xaa \\
\cline { 2 - 5 } & $0 x f f$ & 0x55 & 0x80 & 0xaa \\
\cline { 2 - 5 } & 0x80 & 0x77 & 0xff & 0xbb \\
\hline
\end{tabular}

Table 1 show the values that the PORTx data register be used to processing of data signals for the lighting control on LSDM. The data is to set the direction and output of the input and output data depending on the settings of the DDRx and the PORTx. The PORTx register is stored the value of the logical output port, and verify of DDRxn bit setting contents of the PORTx register. Also, it is designed to load the status value of the corresponding port pin using the PINxn (Port Input Pins Register) bit register. In this case, the PINxn register bits are synchronization in order to optimize the input and output values that are able to avoid intermediate situation lasting phenomenon that the 0 or 1 is not. The the DDRx register value is set to $0 \mathrm{xff}$ for use as the row-by-row lighting control data with the PORTx register output as bit-by-bit. The control logic behavior is cut off the clock supply using sleep mode individually, provide a separate clock to each part to reduce the power consumption in the timer/counter part.

Figure 4 shows the default setting values of the XDIV for the control of demultiply. A clock source that used into the LSDM lighting control logic is $16 \mathrm{MHz}$. Therefore, a clock source is set the value to be activated clock frequency, set to match the control program during behavior of the control logic. A frequency is setting divide to 128 for used of the control logic.

In this paper, the LSDM control algorithm to be used in the LSDM lighting control logic is designed to controlled using the LSDM appropriate to configured with low cost. Figure 5 show the control algorithm to control the behavior state of the LSDM by a pre-set time schedule. The control algorithm flow for the LSDM control is define the type of I/O data, and transmit to the MCU using the data bus by started of illuminance measurement and motion detection through installed sensor on lighting device. The control system from MCU be calculate the illuminated level depending on the set control method by correction of the I/O data, and decide whether to the level maintain by compare the results. The LSDM control algorithm is determine whether to adjust level by comparing with existing measurements value with the data according to the illumination measurement and motion detection. Therefore the lighting control system was applied the control algorithm for an efficient LSDM control.

\begin{tabular}{lcccccccc|c} 
Bit & 7 & 6 & 5 & 4 & 3 & 2 & 1 & 0 \\
\cline { 2 - 7 } & XDIVEN & XDIV6 & XDIV5 & XDIV4 & XDIV3 & XDIV2 & XDIV1 & XDIV0 & XDIV \\
\cline { 2 - 7 } Read/write & $\mathrm{f} / \mathrm{w}$ & $\mathrm{f} / \mathrm{w}$ & $\mathrm{r} / \mathrm{w}$ & $\mathrm{r} / \mathrm{w}$ & $\mathrm{r} / \mathrm{w}$ & $\mathrm{r} / \mathrm{w}$ & $\mathrm{r} / \mathrm{w}$ & $\mathrm{r} / \mathrm{w}$ \\
Initial value & 1 & 0 & 0 & 0 & 0 & 1 & 0 & 0
\end{tabular}

Figure 4. The XDIV default setting value 


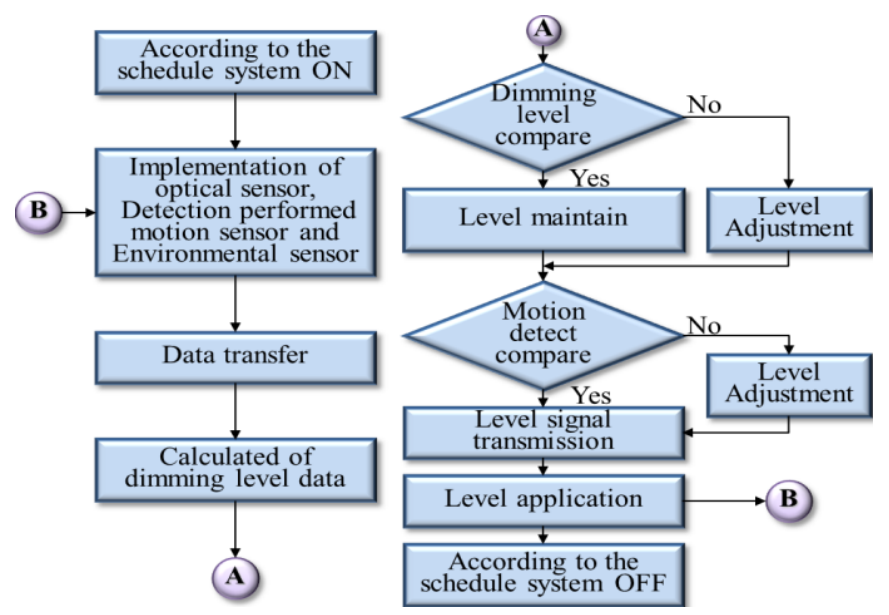

Figure 5. LSDM control algorithm

\section{III.THE EXPERIMENTAL RESULT}

In this paper, a proposed LSDM lighting control logic was measure to power loss rate of the control logic about lighting status that checked the control status using LSDM lighting control data value of input and output from lighting control system. The Lighting control data confirmed data input for LSDM control that apply to the change at any time by user needs. The existing LSDM lighting control logic is replace the street lighting device from the lighting method using sodium lamps and metal halide lamps to LSDM. In addition, the used LED module does not require MCU, because to light up by placing as the line forms or an array form. It is lighting control as the method from only the implementation of the simple the on/off function by measuring the illuminance. For that reason, the street lighting will occurred case to lighting as the excessive brightness by compare to the illuminance of the surrounding with the unnecessary power consuming. The proposed LSDM lighting control logic is lighting control based on factors such as the temperature, humidity, illuminance, vehicle and pedestrian traffic around streetlights, because used the MCU so that we can adjust the brightness using PWM (Pulse Width Modulation) or change the lighting pattern by placing the LED module in the form of a matrix.

In this paper, we are designed control logic for control to the LSDM of efficient lighting control system, and the control program was written for operate in device driver and LSDM. The drivers and written programs were preferences using minicom and RS232C communication through the serial port in order to download to lighting control system. In addition, it was set up environment of the host PC and tftp (trivial file transfer protocol) and transmits. We were used for the cross compiler, because the compiler should behave differently into the host PC and the generated executable file behavior system. The boot loader were compiled for configure the lighting control system, and the download. Also, we were compile with the kernel, the file system, the device drivers, and were to create a lighting control program. The host PC was checks the status of lighting control by download the program into the lighting control system through ethernet or serial.

Figure 6 show the experimental environment. the host PC and the lighting control system were serial connection, and it were configure so that can be control behavior of LSDM through the network on client PC in other places.

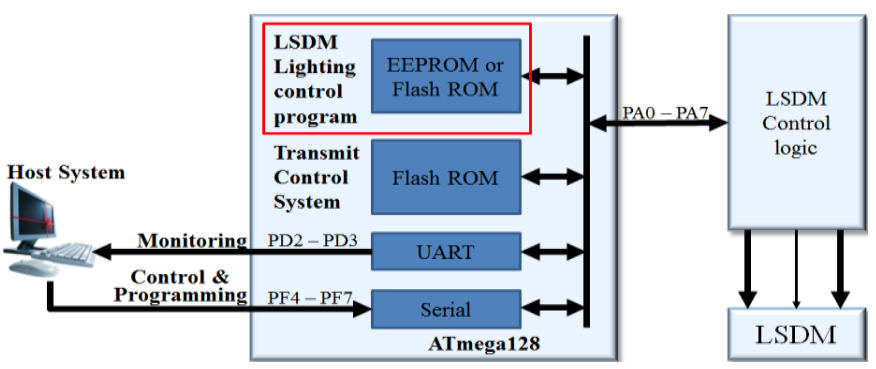

Figure 6. Experiment environment

Figure 7 shows the timing diagram of the lighting control data of the LSDM lighting control logic entered through PORTx of the ATmega128 as apply of the LSDM lighting control algorithm. The lighting control data represents the value of the $\sim$ row[1] in the register-values for the control signals of Table 1 , and the data were entered to sequentially from $\sim$ row $[1]$ to $\sim$ row [4].

Figure 8 was show the time chart of the experimental results on the LSDM lighting control logic operation state of lighting control system that based on the control data entered from the ATmega128 by the LSDM control algorithm. The ATmega128 is enter clock signal to LSDM for data input and display, it entered the data through the PORTx for LSDM lighting.

Figure 9 is a graph showing measured results of the power loss rate by compare to the footprint and the efficiency. A measured result of the power loss rate average value, the power loss is decreased when grows the footprint, and it was consumed the average $2.53 \mathrm{~W}$. The result of comparison to the power loss rate of control logic was reduced by $61.19 \%$.

Figure 10 show the measured results of efficiency and the duty ratio for LSDM operation state by compare to input voltage when applied the LSDM lighting control algorithm. The efficiency of the apply algorithm were could be obtained an average efficiency of $92.03 \%$. The duty cycle were could be obtained an average duty cycle of $54.18 \%$.

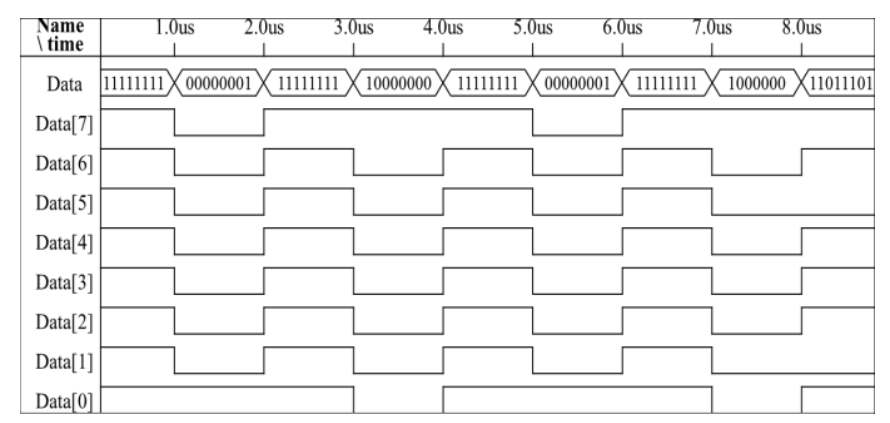

Figure 7. LSDM control data

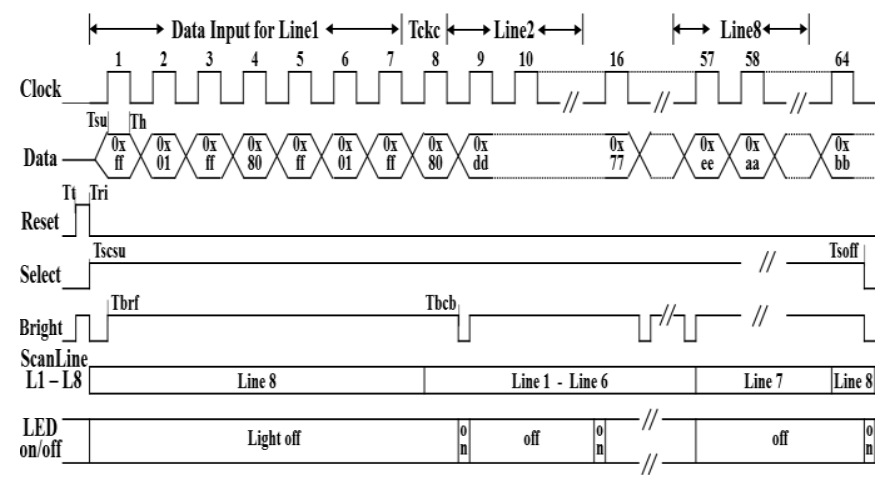

Figure 8. LSDM operation timing chart 


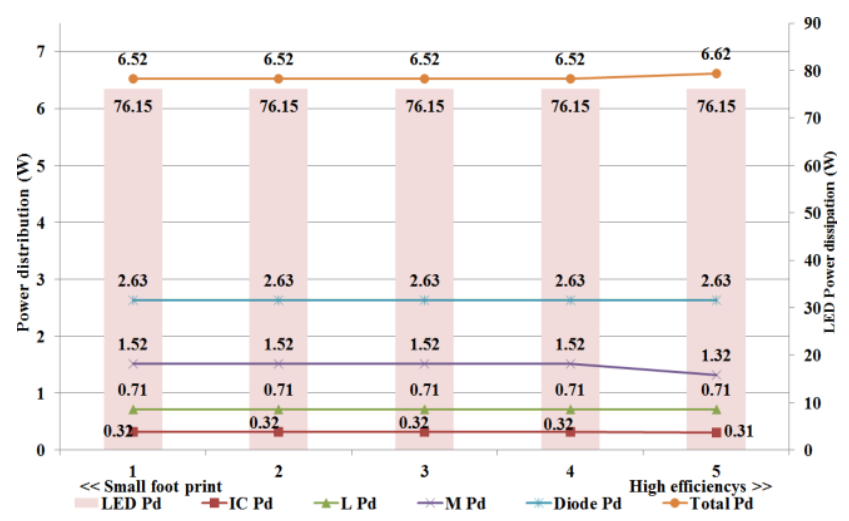

(a) Power dissipation existing lighting control logic

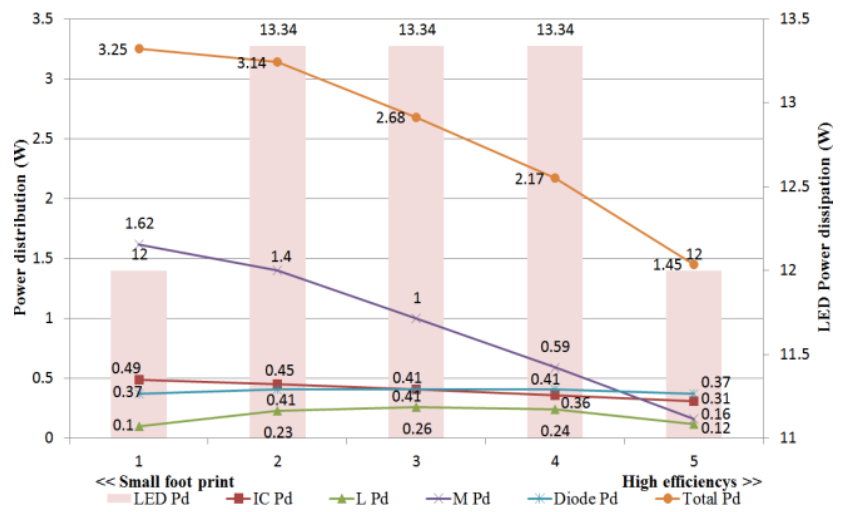

(b) Power dissipation proposed lighting control logic

Figure 9. Power dissipation

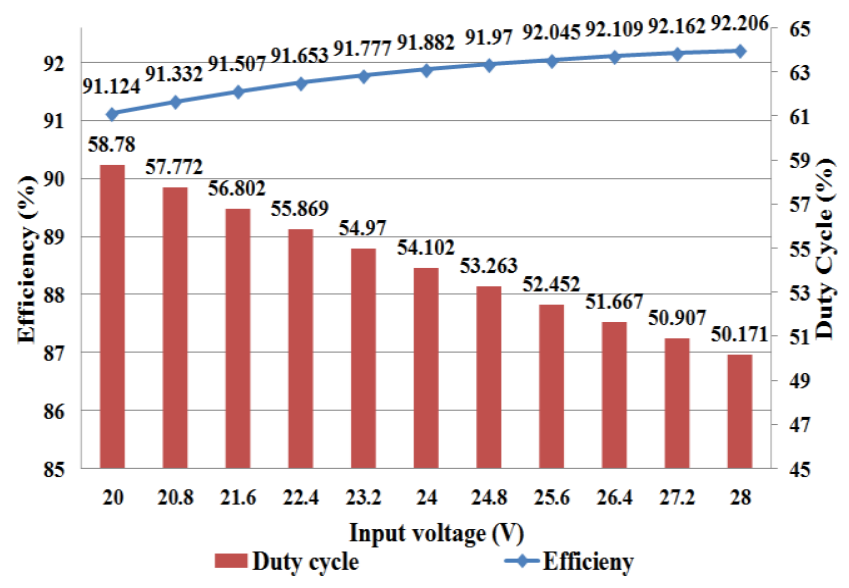

(a) Existing lighting control logic

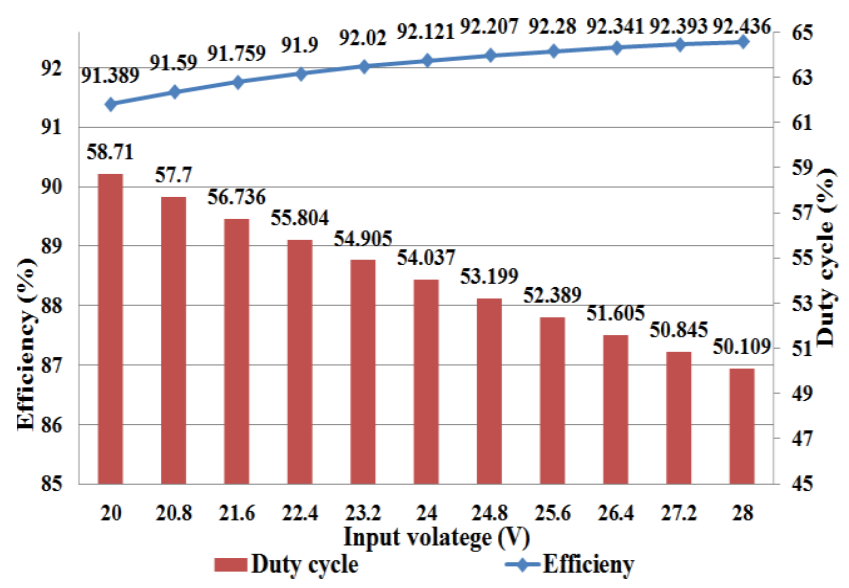

(b) Proposed lighting control logic

Figure 10. Efficiency and duty cycle

\section{IV.CONCLUSION}

In this paper, we proposed an efficient LSDM lighting control logic design on lighting control system using LSDM control algorithm. In this paper, a proposed LSDM lighting control logic were measured to power loss rate of the control logic about LSDM lighting status by download the LSDM control algorithm into the control logic through serial port. LSDM control algorithm is able to control efficiently the lighting control system by the lighting control signal data value of input and output into LSDM lighting control logic.

As a measurement result by check the lighting control status, we were analyzed results on measured the efficiency and the duty ratio for the input power. The efficiency of the proposed LSDM lighting control logic was could be obtained an average efficiency of $92.03 \%$ by measured to increasing $1.14 \%$. The duty cycle were could be obtained an average duty cycle of $54.18 \%$ by measured to decreasing $14.65 \%$. As a result, proposed LSDM lighting control logic than the existing control logic was proved to be effective to improve the overall efficiency of the lighting control system.

The LSDM lighting control logic based on MCU is possible to utilize to be able to lighting control according to the situation by the elements of many real-time monitoring when configuring the sensor network using zigbee communication method.

\section{ACKNOWLEDGMENT}

This work was sponsored by ETRI SW-SoC R\&BD Center, Human Resource Development Project.

\section{REFERENCE}

[1] Seoul Semiconductor, Z-POWER LE D Series Technical Data sheet for W4218X, www.zled.com, 2007.

[2] Sang-Bin Song, In-Seon Yeo, "The Thermal and Circuits Design of an LED Bulb Considering Temperature Property" , Institute of Electrical Engineers, Vol 56, No 7, pp.1261-1267, 2007.

[3] Mi-Young Lee, Woo-Hee Lee, Jun-Ha Lee, Hoong-Joo Lee, "Design of LED Driving Circuits to Detect Degradation Characteristics" , The Korea Institute of Power Electronics, Power Electronics Conference, pp.81-83, July. 2005.

[4] Jung Kwang-Sung, Kim Chang-Beom, Moon Cheol-Hong, "LED Control Board Design using Xilinx Spartan3 FPGA Module", KIEE, CICS '09, pp. 331-332, 2009.

[5] H. Broeck, G. Sauerlander, and M. Vendt, "Power Driver Topologies and Control Schemes for LEDs", Proc. IEEE. APEC, pp. 1319-1325, 2007.

[6] Y. Hu and M. Jovanovic, "LED Driver With Self-Adaptive Drive Voltage", IEEE, Trans. on Power Elec, vol 23, no. 6, pp. 3116-3125, 2008.

[7] Soo-Bin Han, Suck-In Park, Eugine Song, Hak-Guen Jeoung, Bong-Man Jung, Gue-Duk Kim, "Overview of Classification and Characteristics of Recent LED drive IC", KIEE AutumnAnnualConference, pp. 105-107, 2008.

[8] Borbély, A., A. Sámson, and J. Schanda. "The Concept of Correlated Color Temperature Revisited", Color Research \& Application, vol 26, no. 6, pp.450-457, 2001. 